\title{
HETEROSIS AND COMBINING ABILITY FOR SOME TRAITS OF INTRASPECIFIC AND INTERSPECIFIC HYBRIDIZATION BETWEEN SOLANUM MELONGENA AND SOLANUM MACROCARPON
}

\author{
M. S. Hamada ${ }^{\text {; }}$ A. F. Hamaiel${ }^{2}$; S. M. Farid ${ }^{3}$ and M. M. EI- \\ Kady ${ }^{3}$ \\ 1- Dept. of Genetics, Fac. of Agric., Damietta University. 2- Vegetables \\ and Floriculture. Dept., Fac. of Agric., Damietta University. 3-3- \\ Horticultural Res. Institute, Agric. Res. Centre, Giza, Egypt
}

\begin{abstract}
Four parents and their $12 \mathrm{~F}_{1}$ diallel crosses, involving three parents belonging to the species Solanum melongena $\mathrm{L}$. and one parent belonging to species Solanum macrocarpon L. were used. For heterotic effect, heterosis over mid-parents and better parent were detected in many traits, viz.; plant height $(\mathrm{kg})$, number of branches per plant, and total chlorophyll. Also, heterosis over mid-parents and better parent were detected in early fruit yield per plot $(\mathrm{kg})$, early fruit number per plot and total fruit yield ton/fed. For chemical traits heterosis over mid-parents and better parent were detected in total carotene, anthocyanin content, total nitrogen content, total phosphorus, potassium content and total iron traits. The magnitude of GCA variance was greater than SCA variance suggesting the predominance of additive gene action for most studied traits. Among the varieties, the good general combiner was Balady Dark Long $\left(\mathrm{P}_{3}\right)$ for plant height, number of branches per plant, early fruit number per plot, total fruit yield ton/fed and total phosphorus, Balady white Long $\left(\mathrm{P}_{2}\right)$ for total chlorophyll, total nitrogen content, potassium content and total iron. Among the varieties, Balady Dark Round $\left(P_{1}\right)$ was the best for early fruit yield per plot and anthocyanin content, In the case of total carotene Gboma $\left(\mathrm{P}_{4}\right)$ was the best general combiner. The estimates of specific combining ability effects (SCA) showed superior specific combinations, $\left(\mathrm{P}_{2} \times \mathrm{P}_{3}\right)$ for plant height, total fruit yield ton/fed and total phosphorus and $\left(\mathrm{P}_{1} \times \mathrm{P}_{2}\right)$ for number of branches per plant, early fruit yield per plot, early fruit number per plot and total iron. While, $\left(P_{1} \times P_{4}\right)$ was the best for total chlorophyll, total carotene and total nitrogen content, $\left(P_{2} \times P_{4}\right)$ for potassium content. Regarding, reciprocal effect (rij) the results revealed that highest values recorded for $\left(\mathrm{P}_{4} \times \mathrm{P}_{1}\right)$ in the case of plant height $(\mathrm{cm})$, total chlorophyll, total carotene and total iron. While, the hybrid $\left(P_{3} \times P_{1}\right)$ was the best for number of branches, early fruit yield per plot $(\mathrm{kg})$, early fruit number per plot and anthocyanin content and the hybrid $\left(\mathrm{P}_{4} \times \mathrm{P}_{2}\right)$ for total fruit yield(ton/fed) and total phosphorus, the hybrid $\left(P_{4} \times P_{3}\right)$ for total nitrogen content and the hybrid $\left(\mathrm{P}_{3} \times \mathrm{P}_{2}\right)$ was the best for potassium content. Therefore, from general and specific combining ability and some genetic parameters suggested the importance of heterosis breeding for effective utilization of additive genetic variances which had the main role for the improvement of the studied traits in eggplant crop.
\end{abstract}




\section{INTRODUCTION}

Eggplant (Solanum melongena L.), also known as brinjal, aubergine or Guinea squash, is a non-tuberous species of the family Solanaceae. Other cultivated species of eggplants are known as scarlet eggplant (S. aethiopicum L.) and gboma eggplant ( $S$. macrocarpon L.). Cultivated eggplants and their wild relatives belong to the subgenus Leptostenomum (Dunal) Bitter, which includes more than 450 species distributed among 22 sections (Van Eck and Snyder 2006). Studies on interspecific relation and hybridization are of great significance because sometimes interspecific hybridization may be necessary to incorporate desirable genes to cultivated species. Interspecific hybridization via sexual crosses has illustrated various degrees of limitation to crossability, possibly due to sexual barriers Collonnier et al. (2001); Behera and Singh (2002). Hybridization experiments showed that Solanum melongena was crossable with several species of the same subgenus Leptostemonum Collonnier et al. (2001). Interspecific hybrids were produced from crosses between different genotypes of Solanum melongena with Solanum macrocarpon and Solanum torvum. Heterosis breeding as a tool for genetic improvement in eggplant has been studied by several researchers. Heterosis over better parent was detected by Chowdhury et al. (2010) for plant height and fruit yield; Patil et al.(2011) for number of branches per plant; Makani et al. (2013) studied heterosis over mid and better parent for plant height and fruit yield; Chowdhury et al. (2010) for early fruit number and yield.

Combining ability analysis helps in identifying of good combining parents and superior $F_{1}$ crosses. So many studies on eggplant were made for general combining ability (GCA) and specific combining ability (SCA) effects by Babu et al. (2000) for plant height; Salama et al. (2005) for plant height, number of primary branches per plant, early yield, total yield; Kumar et al.(2012) for plant height, number of primary branches and content of anthocyanins.

Rreciprocal effect (rij) analysis helps to identify if there is cytoplasmic or maternal effects or not. expression of reciprocal in eggplant was detected by Brinda and Sivasubramanian(1993); Babu et al. (2000).

Therefore this investigation aimed to produce $F_{1}$ intraspecific and interspecific hybrids from hybridization between Egyptian local cultivars of eggplant speciese (Solanum melongena L.) and African eggplant (Solanum macrocarpon). Present further information dealing with determining of heterosis, general and specific combining ability and their interaction and reciprocal effect over two seasons. 


\section{MATERIALS AND METHODS}

The experiment work was conducted at private farm in Kafr Saad, Damietta Governorate during the period from 2013 to 2015. The genetic materials used in this study were Balady Dark Round $\left(P_{1}\right)$, Balady white Long $\left(P_{2}\right)$, Balady Dark Long $\left(P_{3}\right)$ and Gboma $\left(P_{4}\right)$. Seeds were obtained from The Vegetable Research Institute, Agricultural Research Center (A.R.C), Egypt.

Table 1: The origin and description of the varieties for important traits

\begin{tabular}{|l|l|l|l|}
\hline Var. & Origin & Size and shape of fruit & Color of fruits \\
\hline$P_{1}$ & A.R.E & Large round & Dark purple \\
\hline$P_{2}$ & A.R.E & Long sylenderical & White \\
\hline$P_{3}$ & A.R.E & Long sylenderical & Dark purple \\
\hline$P_{4}$ & West Africa & Small round & Yellow \\
\hline
\end{tabular}

In 2013 growing season (summer), the four parental varieties were crossed according to a complete diallel crosses mating design to obtain six $F_{1}$ hybrids and their corresponding six $F_{1 r}$ (reciprocal) hybrids. In addition, the four parents were also self-pollinated to obtain enough seeds from each parental variety. In the growing season of 2014 and 2015, all the 16 genotypes which included the four parents, the six $F_{1}$ hybrids and six $F_{1 r}$ (reciprocal) hybrids were evaluated in a field trail experiment. The experimental design was the randomized complete blocks design (RCBD) with three replications. Each block consisted of 16 plots. All the genotypes were randomly distributed in each block. Each plot was represented by a single row $4 \mathrm{~m}$ apart to insure 10 plants per row. All agricultural practices were carried out as recommended for eggplant plantation according to the Egyptian Ministry of Agriculture recommendations.

Data were recorded on five plants randomly chosen per plot for all entries for the following traits: plant height in centimeters, number of branches per plant, total chlorophyll $(\mathrm{mg})$, early fruit yield per plot $(\mathrm{kg})$, early fruit number per plot, total fruits yield(ton/fed), total carotene, anthocyanin content, total nitrogen content, total phosphorus content, potassium content and total iron.

Data were calculated and statistically analyzed as outlined by Cochran and Cox (1957). Heterosis was estimated as a percent increase or decrease of $F_{1}$ performance from the mid-parents (m.p) and better parent (p.b). General combining ability (GCA) and specific combining ability (SCA) were analyzed according to the method of Griffing (1956). 


\section{Mean performance}

\section{RESULTS AND DISCUSSION}

The performances of the parental varieties versus their hybrids $\left(F_{1}\right.$ and $\left.F_{1 r}\right)$ are presented in Tables 2 and 3 . The results illustrated that the parental genotype Balady dark long $\left(P_{3}\right)$ showed the highest means for plant height, number of branches, early fruit yield per plot $(\mathrm{kg})$, early fruit number, total fruit yield(ton/fed) and total iron (61.63, $8.27,6.89,115.5,44.93$ and 3.995 , respectively). While, the parental genotype Balady white long $\left(\mathrm{P}_{2}\right)$ showed the highest means for total chlorophyll and total nitrogen content (2.48 and 3.6234), the parental genotype Balady dark round $\left(P_{1}\right)$ for anthocyanin content and potassium content (14.3102 and 4.0617) and the parental genotype Gboma $\left(\mathrm{P}_{4}\right)$ obtained the highest means for total carotene and total phosphorus (0.0215 and 0.06034).

Through the hybrids the results showed that the hybrid $\left(P_{3} \times P_{1}\right)$ obtained the highest means for plant height $(\mathrm{cm})$ and total fruit yield(ton/fed) with values (60.73 and 58.03). While, the hybrid $\left(P_{1} \times P_{3}\right)$ showed the highest means for number of branches, early fruit yield $(\mathrm{kg})$ and anthocyanin content (8.93, 9.40 and 15.6907, respectively), However, the hybrid $\left(\mathrm{P}_{2} \times \mathrm{P}_{3}\right)$ was the best for early fruit number and potassium content (107.83 and 4.145). While, the hybrids $\left(P_{3} \times P_{2}\right),\left(P_{4}\right.$ $\left.\times P_{1}\right),\left(P_{4} \times P_{2}\right),\left(P_{4} \times P_{3}\right)$ and $\left(P_{2} \times P_{1}\right)$ obtained the highest means for: total chlorophyll, total carotene, total nitrogen content, total phosphorus and total iron, respectively $(2.2124,0.0248,3.735,0.0993$ and 4.1033 , respectively).

\section{Heterosis degree}

Data presented in Table 4 showed that the hybrid $\left(\mathrm{P}_{2} \times \mathrm{P}_{1}\right)$ exhibited positive and highly significant heterosis values relative to their mid-parents and better parent for plant height, number of branches, early fruit yield and total fruit yield(ton/fed). While, the hybrid $\left(P_{1} \times P_{4}\right)$ had the largest heterosis values relative to their mid-parents and $\left(P_{1} \times P_{3}\right)$ relative to their better parent for total chlorophyll and also the same hybrid $\left(\mathrm{P}_{1} \times \mathrm{P}_{3}\right)$ relative to their mid-parents had the largest heterosis value for early fruit number and $\left(\mathrm{P}_{2} \times \mathrm{P}_{1}\right)$ relative to their better parent for the same trait. These results were in agreement with those of Chowdhury et al. (2010) who showed heterosis over better parent for fruit yield and Makani et al. (2013) who studied heterosis over midparents and better parent for yield and its components in eggplant.

Obtained data from Table 5 showed that the hybrid $\left(\mathrm{P}_{3} \times \mathrm{P}_{2}\right)$ exhibited positive and highly significant heterosis values relative to their mid-parents and better parent for total carotene, $\left(P_{1} \times P_{3}\right)$ for anthocyanin content, $\left(\mathrm{P}_{3} \mathrm{XP}_{4}\right)$ for total nitrogen content, $\left(\mathrm{P}_{3} \times \mathrm{P}_{1}\right)$ for total 
phosphorus, and $\left(\mathrm{P}_{1} \times \mathrm{P}_{4}\right)$ for total iron. In case of potassium content the hybrid $\left(\mathrm{P}_{4} \mathrm{XP}_{3}\right)$ relative to their mid-parents and the hybrid $\left(\mathrm{P}_{2} \mathrm{xP}_{3}\right)$ relative to their better parent had the best heterosis values.

\section{Combining ability and reciprocal effects}

Results of the analysis of variance for combinig ability (Tables 6 and 7) revealed that GCA, SCA and reciprocal were significant or highly significant for most of the studied traits indicating the importance of both additive and non-additive genetic variance and the cytoplasmic factors which play a major role in the genetic expression of these traits. On the other hand, the magnitude of GCA variance was higher than that of SCA variance for all the characters except total nitrogen content and total phosphorus indicating the preponderance of additive gene action for their genetic control. Furthermore, the interaction of GCA with seasons and reciprocal with seasons was not significant for most studied traits which indicated that these parameters are highly stable with different environments. While, SCA was significant or highly significant for most studied traits which indicated that this parameter is unstable with different environments. The results were in conformity with Babu et al. (2000), Salama et al. (2005) and Sharaf Uddin et al. (2015) who reported that GCA variance was higher than that of SCA variance for all the studied traits indicating that additive gene action was predominant and play the main role in the inheritance of traits under study.

The estimates of GCA effects provide a measure of general combining ability of each parental genotype, thus aids in selection of superior parents for breeding programs. The estimates of general combining ability effect (gi) for each parental genotype for all studied traits are shown in Tables 8 and 9. The obtained data revealed that, none of the parents was the best general combiner for all traits. Among, parental genotypes, the good general combiner was Balady Dark Long $\left(\mathrm{P}_{3}\right)$ for plant height, number of branches, early fruit number, total fruit yield(ton/fed) and total phosphorus. However, Balady white Long $\left(P_{2}\right)$ was the good combiner for total chlorophyll, total nitrogen content, potassium content and total iron and Balady Dark Round $\left(P_{1}\right)$ for early fruit yield $(\mathrm{kg})$ and anthocyanin content, the parental genotype Gboma $\left(\mathrm{P}_{4}\right)$ was the best for total carotene. Similar results were obtained by Babu et al. (2000) and Salama et al. (2005).

The estimates of specific combining ability effects (Tables 10 and 11) showed that, the hybrid $\left(\mathrm{P}_{2} \mathrm{XP}_{3}\right)$ exhibited positive and highly significant specific combining ability for plant height, total fruit yield(ton/fed) and total phosphorus (5.46, 22.45 and 0.0177, respectively). The hybrid $\left(\mathrm{P}_{1} \times \mathrm{P}_{2}\right)$ was good specific combiner for number of branches, early fruit yield, early fruit number and total iron 
(0.9438, 2.0629, 13.4896 and 0.8226 , respectively). For total chlorophyll, total carotene and total nitrogen content traits $\left(\mathrm{P}_{1} \mathrm{xP}_{4}\right)$ exhibited positive and highly significant specific combining ability with values $\left(0.1206,0.0046\right.$ and 0.3712 , respectively), the hybrid $\left(P_{1} \times P_{3}\right)$ was the best one for anthocyanin content (4.167) and $\left(\mathrm{P}_{2} \times \mathrm{PP}_{4}\right)$ for potassium content trait (0.1389).

Although these hybrids were not the superior but it obtained positive highly significant or significant specific combining ability: $\left(\mathrm{P}_{1} \mathrm{XP}_{2}\right)$ for plant height with value 4.7625, $\left(\mathrm{P}_{1} \mathrm{x} \mathrm{P}_{3}\right)$ for plant height, number of branches and early fruit yield $(2.0167,0.6896$ and 1.086), $\left(\mathrm{P}_{2} \mathrm{XP}_{3}\right)$ for total chlorophyll, total carotene and total iron $(0.065,0.0035$ and 0.1668), $\left(\mathrm{P}_{2} \mathrm{XP}_{4}\right)$ for total carotene, anthocyanin content, total nitrogen content and total phosphorus $(0.0003,2.6965,0.1737$ and 0.0002 , respectively), $\left(P_{1} \times P_{4}\right)$ for total iron 0.3876 and $\left(P_{3} \times P_{4}\right)$ for total carotene, total nitrogen content and total phosphorus $(0.0016,0.2345$ and 0.0062 , respectively).

Regarding, reciprocal effect (rij) the results revealed that highest positive values recorded for $\left(\mathrm{P}_{4} \mathrm{XP}_{1}\right)$ in the case of plant height, total chlorophyll, total carotene and total iron with values $(0.2,0.1187$, 0.0002 and 1.5683, respectively), the hybrid $\left(P_{3} \times P_{1}\right)$ was the best for number of branches, early fruit yield, early fruit number and anthocyanin content $(0.4833,1.8784,26.1667$ and 1.1843, respectively). Also the same hybrid $\left(P_{3} \times P_{1}\right)$ obtained positive highly significant specific combining ability for total chlorophyll and total iron (0.1083 and 0.4383). While, the hybrid $\left(\mathrm{P}_{4} \times \mathrm{P}_{2}\right)$ was the highest for total fruit yield(ton/fed) and total phosphorus (1.745 and 0.0066, respectively) and $\left(\mathrm{P}_{4} \times \mathrm{P}_{3}\right)$ for total nitrogen content trait with value (0.3417), also the hybrid $\left(\mathrm{P}_{4} \times \mathrm{P}_{2}\right)$ obtained positive highly significant specific combining ability for number of branches and total iron (0.2667 and 0.5825$)$ and $\left(P_{2} \times P_{1}\right)$ for total nitrogen content with value 0.1258 . These results were in agreement with those of Babu et al. (2000) and Salama et al. (2005).

In conclusion, the results obtained from general and specific combining ability and some genetic parameters indicated the importance of heterosis breeding for effective utilization of additive genetic variances which had the main role for the improvement of the studied traits in eggplant crop. 
Table 2: Mean performance of parental genotypes and their hybrids for yield and its components in eggplant

\begin{tabular}{|c|c|c|c|c|c|c|}
\hline $\begin{array}{l}\text { Genotype } \\
\mathrm{s}\end{array}$ & $\begin{array}{l}\text { Plant } \\
\text { height }(\mathrm{cm})\end{array}$ & $\begin{array}{ll}\begin{array}{l}\text { Number } \\
\text { branches }\end{array} & \text { of } \\
\end{array}$ & $\begin{array}{l}\text { Total } \\
\text { chlorophyll }\end{array}$ & $\begin{array}{l}\text { Early fruit } \\
\text { yield(kg) }\end{array}$ & $\begin{array}{l}\text { Early fruit } \\
\text { number }\end{array}$ & $\begin{array}{l}\text { Total fruit } \\
\text { yield(ton/fed) }\end{array}$ \\
\hline $\mathbf{P}_{1}$ & 57.13 & 7.27 & 2.081 & 4.83 & 37.33 & 41.69 \\
\hline $\mathbf{P}_{2}$ & 53.4 & 6.5 & 2.48 & 5.39 & 97.17 & 33.48 \\
\hline $\mathbf{P}_{3}$ & 61.63 & 8.27 & 2.1494 & 6.89 & 115.5 & 44.93 \\
\hline $\mathbf{P}_{4}$ & 28.23 & 4.8 & 1.7315 & 0 & 0 & 13.04 \\
\hline \multicolumn{7}{|l|}{ Crosses } \\
\hline$P_{1} \times P_{2}$ & 58.63 & 8 & 2.0902 & 7.77 & 80.67 & 52.67 \\
\hline$P_{1} \times P_{3}$ & 58.77 & 8.93 & 2.1949 & 9.40 & 105.67 & 52.44 \\
\hline $\mathbf{P}_{1} \times \mathbf{P}_{4}$ & 21.27 & 3.43 & 2.0329 & 0 & 0 & 1.05 \\
\hline $\mathbf{P}_{\mathbf{2}} \times \mathbf{P}_{\mathbf{1}}$ & 58.77 & 8.13 & 1.945 & 8.87 & 91.17 & 56.57 \\
\hline $\mathbf{P}_{2} \times \mathbf{P}_{3}$ & 58.7 & 7.27 & 2.2103 & 6.55 & 107.83 & 44.71 \\
\hline $\mathrm{P}_{2} \times \mathrm{P}_{4}$ & 10.3 & 3.17 & 1.6552 & 0 & 0 & 0.76 \\
\hline$P_{3} \times P_{1}$ & 60.73 & 7.97 & 1.9782 & 5.64 & 53.33 & 58.03 \\
\hline $\mathbf{P}_{3} \times \mathbf{P}_{\mathbf{2}}$ & 60.53 & 7.17 & 2.2124 & 4.84 & 88.5 & 49.51 \\
\hline $\mathbf{P}_{3} \times \mathbf{P}_{4}$ & 12.4 & 3.03 & 1.6183 & 0 & 0 & 2.09 \\
\hline $\mathbf{P}_{4} \times \mathbf{P}_{1}$ & 20.87 & 3.37 & 1.7955 & 0 & 0 & 1.83 \\
\hline $\mathrm{P}_{4} \times \mathrm{P}_{2}$ & 10.97 & 2.63 & 1.6384 & 0 & 0 & 0.63 \\
\hline $\mathbf{P}_{4} \times \mathbf{P}_{3}$ & 20.67 & 3.57 & 1.7067 & 0 & 0 & 1.89 \\
\hline
\end{tabular}

Table 3: Mean performance of parental genotypes and their hybrids for chemical traits in eggplant

\begin{tabular}{|c|c|c|c|c|c|c|}
\hline $\begin{array}{l}\text { Genotype } \\
\text { s }\end{array}$ & $\begin{array}{l}\text { Total } \\
\text { carotene }\end{array}$ & $\begin{array}{l}\text { Anthocyanin } \\
\text { content }\end{array}$ & $\begin{array}{c}\text { Total nitrogen } \\
\text { content }\end{array}$ & $\begin{array}{l}\text { Total } \\
\text { phosphorus }\end{array}$ & $\begin{array}{c}\text { Potassium } \\
\text { content }\end{array}$ & Total iron \\
\hline $\mathbf{P}_{1}$ & 0.01067 & 14.3102 & 3.0017 & 0.053835 & 4.0617 & 1.9783 \\
\hline $\mathbf{P}_{2}$ & 0.00683 & 0.2309 & 3.6234 & 0.056335 & 3.99 & 2.9667 \\
\hline $\mathbf{P}_{3}$ & 0.01167 & 8.754 & 2.5267 & 0.049835 & 3.57 & 3.995 \\
\hline $\mathbf{P}_{4}$ & 0.0215 & 0.3714 & 2.3317 & 0.06034 & 2.45 & 1.3234 \\
\hline \multicolumn{7}{|l|}{ Crosses } \\
\hline $\mathbf{P}_{1} \times \mathbf{P}_{2}$ & 0.00917 & 1.2009 & 2.945 & 0.0485 & 3.0217 & 4.045 \\
\hline $\mathbf{P}_{1} \times \mathbf{P}_{3}$ & 0.011 & 15.6907 & 2.5617 & 0.044 & 3.5467 & 3.4384 \\
\hline$P_{1} \times P_{4}$ & 0.0245 & 0.87 & 3.24 & 0.061 & 3.1234 & 3.5467 \\
\hline$P_{2} \times P_{1}$ & 0.0105 & 1.0447 & 2.6934 & 0.0572 & 3.875 & 4.1033 \\
\hline$P_{2} \times P_{3}$ & 0.01567 & 2.9923 & 3.0017 & 0.0852 & 4.145 & 2.8517 \\
\hline $\mathbf{P}_{2} \times \mathbf{P}_{4}$ & 0.0185 & 0.2642 & 3.0833 & 0.07584 & 3.3467 & 1.7317 \\
\hline$P_{3} \times P_{1}$ & 0.012 & 13.3222 & 3.2883 & 0.0982 & 3.3717 & 2.5617 \\
\hline$P_{3} \times P_{2}$ & 0.01583 & 1.9565 & 2.98 & 0.09467 & 3.5817 & 3.8817 \\
\hline$P_{3} \times P_{4}$ & 0.0225 & 1.3303 & 3.5017 & 0.063 & 2.7 & 0.38 \\
\hline $\mathbf{P}_{4} \times \mathbf{P}_{1}$ & 0.02483 & 0.588 & 3.6467 & 0.07667 & 3.2284 & 0.41 \\
\hline$P_{4} \times P_{2}$ & 0.01967 & 0.4049 & 3.735 & 0.06267 & 3.485 & 0.5667 \\
\hline $\mathbf{P}_{4} \times \mathbf{P}_{3}$ & 0.0235 & 0.489 & 2.8184 & 0.09934 & 3.4917 & 0.545 \\
\hline
\end{tabular}


Table 4: Heterosis relative to the mid parents (m.p) and better parent (b.p) for yield and its components in eggplant hybrids

\begin{tabular}{|c|c|c|c|c|c|c|c|c|c|c|c|c|}
\hline \multirow[t]{2}{*}{ Crosses } & \multicolumn{2}{|c|}{ Plant height(cm) } & \multicolumn{2}{|c|}{ Number of branches } & \multicolumn{2}{|c|}{ Total chlorophyll } & \multicolumn{2}{|c|}{ Early fruit yield(kg) } & \multicolumn{2}{|c|}{ Early fruit number } & \multicolumn{2}{|c|}{ Total fruit yield(ton/fed) } \\
\hline & m.p & b.p & m.p & b.p & m.p & b.p & m.p & b.p & m.p & b.p & m.p & b.p \\
\hline $1 \times 2$ & $6.0918^{* *}$ & $2.6254^{* *}$ & $16.2229^{* *}$ & $10.092^{*}$ & $-8.3469^{* *}$ & $-15.7198^{* *}$ & $52.0068^{* *}$ & $44.1527^{* *}$ & $19.9502^{* *}$ & $-16.9812^{* *}$ & 40.0112 & $26.2287^{* \star}$ \\
\hline $1 \times 3$ & $-1.0390^{* *}$ & $-4.6517^{* *}$ & $15.0219^{* \star}$ & 8.0649 & $3.7668^{* *}$ & 2.1169 & $60.358^{* *}$ & $36.3723^{* *}$ & $38.2766^{* *}$ & $-8.5139^{* *}$ & $21.0866^{* *}$ & $25.7909^{* \star}$ \\
\hline $1 \times 4$ & $-50.1763^{* \star}$ & $-62.7775^{* \star}$ & $-43.0936^{* *}$ & $-52.752^{* \star}$ & $6.6413^{* \star}$ & $-2.3138^{* *}$ & 0 & 0 & 0 & 0 & $-96.1633^{* *}$ & $-97.4815^{\text {*t }}$ \\
\hline $2 \times 1$ & $6.3334^{* \star}$ & $2.8591^{* *}$ & $18.1595^{* *}$ & $11.9264^{* *}$ & $-14.7117^{* *}$ & $-21.5726^{* *}$ & $73.6704^{* *}$ & $64.697^{* *}$ & $35.5636^{* *}$ & $-6.175^{* *}$ & $50.4895^{* *}$ & $35.6756^{*}$ \\
\hline $2 \times 3$ & $2.0572^{* *}$ & $-4.7596^{* *}$ & $-1.5803^{* *}$ & $-12.0968^{* *}$ & $-4.5093^{\text {t* }}$ & $-10.875^{\text {** }}$ & 6.6385 & $-5.0053^{* \star}$ & $1.4109^{* *}$ & $-6.6377^{* *}$ & $14.0416^{\text {** }}$ & $-0.4863^{\text {t* }}$ \\
\hline $2 \times 4$ & $-74.7653^{* \star}$ & $-80.7116^{* \star}$ & $-43.9522^{* *}$ & $-51.2815^{* *}$ & $-21.3986^{* \star}$ & $-33.2601^{* *}$ & 0 & 0 & 0 & 0 & $-96.7244^{* *}$ & $-97.7242^{\star \star}$ \\
\hline $3 \times 1$ & $2.2734^{* *}$ & $-1.4602^{* *}$ & $2.5751^{\star *}$ & $-3.6290^{* *}$ & $-6.4782^{* *}$ & $-7.9652^{* \star}$ & $-3.7397^{* *}$ & $-18.1379^{* *}$ & $-30.2077^{* *}$ & $-53.8242^{* *}$ & $33.9829^{* \star}$ & $29.1528^{* *}$ \\
\hline $3 \times 2$ & $5.245^{* *}$ & $-1.7847^{* *}$ & $-2.9347^{* *}$ & $-13.3065^{* *}$ & $-4.4207^{* *}$ & $-10.7923^{* \star}$ & $-21.1617^{* *}$ & $-29.7701^{* *}$ & $-16.7711^{* \star}$ & $-23.3766^{* *}$ & $26.2869^{* *}$ & $10.1991^{* \star}$ \\
\hline $3 \times 4$ & $-72.4037^{* *}$ & $-79.8811^{* \star}$ & $-53.5711^{\star \star}$ & $-63.3062^{* *}$ & $-16.6007^{* *}$ & $-24.7075^{* *}$ & 0 & 0 & 0 & 0 & $-92.7985^{* *}$ & $-95.3539^{* *}$ \\
\hline $4 \times 1$ & $-51.1123^{* *}$ & $-63.4768^{* \star}$ & $-44.1991^{* *}$ & $-53.6698^{* \star}$ & $-5.8098^{* *}$ & $-13.7194^{* \star}$ & 0 & 0 & 0 & 0 & $-93.3077^{\star *}$ & $-95.607^{* \star}$ \\
\hline $4 \times 2$ & $-73.1311^{* \star}$ & $-79.4625^{* *}$ & $-53.392^{* *}$ & $-59.4869^{* *}$ & $-22.1964^{* *}$ & $-33.9375^{* *}$ & 0 & 0 & 0 & 0 & $-97.3026^{* *}$ & $-98.1259^{* *}$ \\
\hline $4 \times 3$ & $-54.0065^{* *}$ & $-66.4687^{* *}$ & $-45.4083^{* *}$ & $-56.855^{* *}$ & $-12.0476^{* *}$ & $-20.5969^{* *}$ & 0 & 0 & 0 & 0 & $-93.4954^{* *}$ & $-95.8035^{* *}$ \\
\hline $\begin{array}{c}\text { LSD } 0.05 \\
0.01\end{array}$ & $\begin{array}{l}4.2024 \\
5.5892\end{array}$ & $\begin{array}{l}4.8525 \\
6.4538\end{array}$ & $\begin{array}{c}1.497 \\
1.9910\end{array}$ & $\begin{array}{l}1.7286 \\
2.299\end{array}$ & $\begin{array}{l}0.4074 \\
0.5419\end{array}$ & $\begin{array}{l}0.4704 \\
0.6257\end{array}$ & $\begin{array}{l}1.2482 \\
1.6601\end{array}$ & $\begin{array}{l}1.4413 \\
1.9169\end{array}$ & $\begin{array}{l}18.5946 \\
24.7309\end{array}$ & $\begin{array}{l}21.4712 \\
28.5567\end{array}$ & $\begin{array}{l}5.1379 \\
6.8334 \\
\end{array}$ & $\begin{array}{l}5.9327 \\
7.8905\end{array}$ \\
\hline
\end{tabular}

* Significant at $0.05 \%,{ }^{* *}$ Significant at $0.01 \%$

Table 5: Heterosis relative to the mid parents (m.p) and better parent (b.p) for chemical traits in eggplant hybrids

\begin{tabular}{|c|c|c|c|c|c|c|c|c|c|c|c|c|}
\hline \multirow[t]{2}{*}{ Crosses } & \multicolumn{2}{|c|}{ Total carotene } & \multicolumn{2}{|c|}{ Anthocyanin content } & \multicolumn{2}{|c|}{ Total nitrogen content } & \multicolumn{2}{|c|}{ Total phosphorus } & \multicolumn{2}{|c|}{ Potassium content } & \multicolumn{2}{|c|}{ Total iron } \\
\hline & m.p & b.p & m.p & b.p & m.p & b.p & m.p & b.p & m.p & b.p & m.p & b.p \\
\hline $1 \times 2$ & $4.7627^{* *}$ & $-14.0621^{* *}$ & $-83.4833^{* *}$ & $-91.6084^{* *}$ & $-11.0943^{*+}$ & $-18.7216^{* *}$ & $-11.9543^{* *}$ & $-13.9079^{* *}$ & $-24.9426^{* *}$ & $-25.605^{* *}$ & $63.6013^{\text {t* }}$ & $36.3491^{* *}$ \\
\hline $1 \times 3$ & $-1.4933^{* \star}$ & $-5.7129^{* \star}$ & $36.0614^{\star \star}$ & $9.6474^{* *}$ & $-7.3241^{* *}$ & $-14.6569^{* \star}$ & $-15.1153^{* \star}$ & $-18.2688^{* \star}$ & $-7.0548^{* *}$ & $-12.6807^{* *}$ & $15.124^{\star \star}$ & $-13.9337^{* *}$ \\
\hline $1 \times 4$ & $52.3300^{* *}$ & $13.9535^{\star *}$ & $-88.1483^{* *}$ & $-93.9204^{* \star}$ & $21.4996^{* *}$ & $7.9406^{* *}$ & $6.8582^{* \star}$ & $1.1022^{* *}$ & $-4.0696^{* *}$ & $-23.1024^{* *}$ & $114.8411^{* *}$ & $79.2777^{* *}$ \\
\hline $2 \times 1$ & $19.9966^{* *}$ & $-1.5656^{* \star}$ & $-85.631^{* \star}$ & $-92.6996^{* *}$ & $-18.6913^{\text {t* }}$ & $-25.6669^{* \star}$ & $3.78506^{* \star}$ & $1.4822^{* *}$ & $-3.7470^{* *}$ & $-4.5966^{* *}$ & $65.9592^{* \star}$ & $38.3143^{* *}$ \\
\hline $2 \times 3$ & $69.3676^{* *}$ & $34.2862^{* *}$ & $-33.3923^{* *}$ & $-65.8179^{* \star}$ & -2.3854 & $-17.1582^{* *}$ & $60.4314^{* *}$ & $51.176^{* *}$ & $9.6561^{* \star}$ & 3.8847 & $-18.0755^{* \star}$ & $-28.6195^{* *}$ \\
\hline $2 \times 4$ & $30.5875^{* *}$ & $-13.9535^{* *}$ & $-12.2717^{* *}$ & $-28.8676^{* *}$ & $3.5525^{* *}$ & $-14.9047^{* *}$ & $29.9991^{* *}$ & $25.6899^{* \star}$ & $3.9332^{* \star}$ & $-16.1241^{* *}$ & $-19.2681^{* *}$ & $-41.6278^{* *}$ \\
\hline $3 \times 1$ & $7.4619^{* *}$ & $2.8586^{* *}$ & $15.5230^{* *}$ & $-6.9038^{* *}$ & $18.9624^{* *}$ & $9.5498^{* *}$ & $89.3894^{* *}$ & $82.3535^{* *}$ & $-11.6409^{* *}$ & $-16.9892^{* *}$ & $-14.23^{* *}$ & $-35.8786^{* *}$ \\
\hline $3 \times 2$ & $71.173^{* *}$ & $35.7177^{\star \star}$ & $-56.4489^{* *}$ & $-77.6502^{* \star}$ & $-3.0894^{* *}$ & $-17.7557^{* *}$ & $78.3272^{* *}$ & $68.0394^{* \star}$ & $-5.2474^{* *}$ & $-10.2343^{*+}$ & $11.5152^{* *}$ & $-2.8373^{* *}$ \\
\hline $3 \times 4$ & $35.6791^{* *}$ & $4.6512^{* *}$ & $-70.8439^{* *}$ & $-84.8035^{* \star}$ & $44.1498^{* *}$ & $38.5887^{* *}$ & $14.3687^{* *}$ & $4.4170^{* *}$ & $-10.299^{* *}$ & $-24.3697^{* *}$ & $-85.7099^{* *}$ & $-90.4881^{* *}$ \\
\hline $4 \times 1$ & $54.4036^{* *}$ & $15.5047^{\star \star}$ & $-91.9899^{* *}$ & $-95.891^{* *}$ & $22.4725^{* \star}$ & $21.4882^{* *}$ & $34.2997^{* \star}$ & $27.0656^{* \star}$ & $-0.8446^{* *}$ & $-20.5173^{* *}$ & $\begin{array}{l}-75.1639^{* *} \\
\end{array}$ & $-79.2751^{* *}$ \\
\hline $4 \times 2$ & $38.8251^{* *}$ & $-8.5256^{* *}$ & $34.457^{*+}$ & $9.0211^{* *}$ & $25.4398^{* *}$ & 3.0814 & $7.4227^{7 *+}$ & $3.8618^{* *}$ & $8.2298^{\star \star+}$ & $-12.6566^{* *}$ & $-73.5804^{* *}$ & $-80.8976^{* *}$ \\
\hline $4 \times 3$ & $41.7093^{* *}$ & $9.3023^{* *}$ & $-89.2826^{* *}$ & $-94.414^{* *}$ & $16.0209^{* *}$ & $11.5449^{* *}$ & $80.3304^{* *}$ & $64.6391^{* *}$ & $16.0017^{* \star}$ & $-2.1947^{* *}$ & $\begin{array}{l}-79.5049^{* *} \\
\end{array}$ & $-86.3579^{* *}$ \\
\hline LSD 0.05 & 0.0065 & 0.0075 & 1.8935 & 2.1864 & 0.8525 & 0.9844 & 0.0273 & 0.0315 & 1.1546 & 1.3332 & 1.2711 & 1.4678 \\
\hline 0.01 & 0.0086 & 0.01 & 2.5183 & 2.9079 & 1.1339 & $\begin{array}{l}1.3093 \\
\end{array}$ & 0.0363 & 0.042 & 1.5356 & 1.7731 & 1.6906 & 1.9522 \\
\hline
\end{tabular}

* Significant at $0.05 \%,{ }^{* *}$ Significant at $0.01 \%$

Table 6: Analysis of variance and mean squares for yield and its components of genetic variances in eggplant over two seasons

\begin{tabular}{|c|c|c|c|c|c|c|c|}
\hline $\begin{array}{l}\text { Source of } \\
\text { variation }\end{array}$ & d.f & Plant height(cm) & Number of branches & Total chlorophyll & Early fruit yield(kg) & Early fruit number & Total fruit yield(ton/fed) \\
\hline Hybrids & 15 & $2759.738^{*+*}$ & $31.8549^{* *}$ & $0.3921^{\text {** }}$ & $79.5954^{* *}$ & $13885.91^{*}$ & $5110.404^{* *}$ \\
\hline GCA & 3 & $10187.06^{* *+}$ & $111.6525^{* *}$ & 1.1829 & $302.5267^{* *+}$ & $55158.81^{\text {** }}$ & 13157.86 \\
\hline SCA & 6 & $1767.718^{* *+}$ & $23.0431^{* *}$ & $0.3225^{* *}$ & $38.6015^{* *}$ & $5523.967^{* *}$ & $5813.021^{* *}$ \\
\hline Reciprocal & 6 & $38.0944^{* *}$ & 0.7678 & 0.0662 & $9.1235^{* *}$ & 1611.403 & 384.0589 \\
\hline Seasons & 1 & $82.14^{* *}$ & $2.7338^{*}$ & $0.5426^{* *}$ & 14.1834** & $656.2604^{* *}$ & $513.7158^{* *}$ \\
\hline Hybrids x S & 15 & $37.244^{* *}$ & $1.3293^{*}$ & 0.0746 & $2.5998^{*+1}$ & $749.0604^{* *}$ & $246.8706^{* *}$ \\
\hline GCA XS & 3 & 58.0806 & 2.2903 & $0.2047^{* *}$ & 3.7786 & 639.3403 & $\begin{array}{ll}202.3728 \\
\end{array}$ \\
\hline SCAXS & 6 & $59.2131^{* *}$ & $1.5992^{*}$ & 0.0158 & $1.7239^{* *}$ & $805.0781^{* *}$ & $225.2563^{* *}$ \\
\hline Reci $\times \mathbf{S}$ & 6 & 4.8567 & 0.5789 & 0.0683 & $2.8862^{2 *}$ & $747.9028^{* *}$ & $290.7338^{* *}$ \\
\hline Error & 60 & 4.4138 & 0.5603 & 0.0415 & 0.3895 & 86.4417 & 25.12351 \\
\hline
\end{tabular}

* Significant at $0.05 \%,{ }^{*}$ Significant at $0.01 \%$ levels of probability 
Table 7: Analysis of variance and mean squares for chemical traits of genetic variances in eggplant over two seasons

\begin{tabular}{|c|c|c|c|c|c|c|c|}
\hline $\begin{array}{l}\text { Source of } \\
\text { variation }\end{array}$ & d.f & $\begin{array}{c}\text { Total } \\
\text { carotene }\end{array}$ & $\begin{array}{c}\text { Anthocyanin } \\
\text { content }\end{array}$ & $\begin{array}{l}\text { Total nitrogen } \\
\text { content }\end{array}$ & $\begin{array}{c}\text { Total } \\
\text { phosphorus }\end{array}$ & $\begin{array}{c}\text { Potassium } \\
\text { content }\end{array}$ & Total iron \\
\hline Hybrids & 15 & $0.000219^{\star \star}$ & $187.7526^{\star \star}$ & $1.071922^{\star \star}$ & $0.00197^{\star \star}$ & $1.3157^{\star \star}$ & $11.9668^{\star \star}$ \\
\hline GCA & 3 & $0.000801^{\star *}$ & $655.0564^{\star \star}$ & 0.782763 & 0.00112 & $3.7490^{*}$ & $29.3379^{\star}$ \\
\hline SCA & 6 & $0.000145^{* *}$ & $138.0962^{\star \star}$ & $1.4640^{\star \star}$ & $0.00196^{*}$ & $0.5482^{*}$ & $8.72002^{\star \star}$ \\
\hline Reciprocal & 6 & 0.0000026 & $3.7571^{\star \star}$ & $0.8244^{\star \star}$ & $0.00242^{\star \star}$ & 0.8665 & $6.52799^{\star \star}$ \\
\hline Seasons & 1 & 0.0000034 & $27.3547^{* *}$ & $1.7308^{* *}$ & $0.00079^{*}$ & $4.5632^{* *}$ & $2.6567^{\star}$ \\
\hline Hybrids x S & 15 & 0.0000078 & $6.61698^{\star \star}$ & 0.231022 & $0.00060^{\star \star}$ & 0.4037 & 0.6587 \\
\hline GCA $\times$ S & 3 & 0.0000063 & $20.0468^{*}$ & 0.4332 & 0.0007 & $0.5451^{*}$ & 0.5316 \\
\hline SCA $\times$ S & 6 & 0.00001 & $5.4432^{\star \star}$ & 0.1742 & $0.00083^{\star \star}$ & 0.1841 & $0.9555^{*}$ \\
\hline Reci x S & 6 & 0.0000063 & 1.0759 & 0.1868 & 0.00033 & 0.5527 & 0.4252 \\
\hline Error & 60 & 0.000011 & 0.8964 & 0.181693 & 0.00019 & 0.3333 & 0.40398 \\
\hline
\end{tabular}

${ }^{*}$ Significant at $0.05 \%,{ }^{* \star}$ Significant at $0.01 \%$ levels of probability

Table 8: Estimates of general combining ability effects for parental genotypes for yield and its components over two seasons

\begin{tabular}{|c|c|c|c|c|c|c|}
\hline Parents & Plant height(cm) & Number of branches & Total chlorophyll & Early fruit yield(kg) & Early fruit number & Total fruit yield(ton/fed) \\
\hline P1 & $8.35^{\star *}$ & $0.95208^{\star \star}$ & $0.05484^{* \star}$ & $1.40608^{\star \star}$ & 2.11458 & -4.76261 \\
\hline $\mathrm{P} 2$ & $4.775^{* *}$ & $0.32708^{* *}$ & $0.11895^{* *}$ & $1.08913^{* *}$ & $21.73958^{*}$ & $10.30965^{* *}$ \\
\hline P3 & $8.57083^{* \star}$ & $0.96458^{* *}$ & $0.05745^{* \star}$ & $1.26551^{* *}$ & $24.71875^{\star \star}$ & $15.63857^{* *}$ \\
\hline P4 & $-21.6958^{* \star}$ & $-2.24375^{\star \star}$ & $-0.23124^{*}$ & $-3.76073^{\star *}$ & $-48.5729^{* \star}$ & $-21.1856^{* *}$ \\
\hline S.E(gi) & 0.26261 & 0.09356 & 0.02546 & 0.07801 & 1.16218 & 0.62654 \\
\hline
\end{tabular}

${ }^{*}$ Significant at $0.05 \%,{ }^{* \star}$ Significant at $0.01 \%$ levels of probability

Table 9: Estimates of general combining ability effects for parental genotypes for chemical traits over two seasons

\begin{tabular}{|c|c|c|c|c|c|c|}
\hline Parents & Total carotene & Anthocyanin content & Total nitrogen content & Total phosphorus & Potassium content & Total iron \\
\hline $\mathrm{P} 1$ & $-0.00198^{\star \star}$ & $3.67834^{\star \star}$ & -0.01385 & $-0.00626^{\star \star}$ & $0.09948^{\star \star}$ & $0.3624^{\star \star}$ \\
\hline P2 & $-0.00327^{* *}$ & $-2.94811^{* *}$ & $0.14948^{* *}$ & $-0.00082^{* *}$ & $0.24260^{* *}$ & $0.49385^{* *}$ \\
\hline P3 & $-0.00067^{* \star}$ & $2.67239^{\star *}$ & $-0.16052^{* *}$ & $0.00509^{\star \star}$ & 0.06031 & $0.31073^{\star \star}$ \\
\hline P4 & $0.00592^{* *}$ & $-3.40261^{* *}$ & 0.0249 & $0.00199^{* \star}$ & $-0.4024^{* *}$ & $-1.16698^{* *}$ \\
\hline S.E(gi) & 0.00041 & 0.11835 & 0.05328 & 0.00171 & 0.07216 & 0.07945 \\
\hline
\end{tabular}

* Significant at $0.05 \%,{ }^{* \star}$ Significant at $0.01 \%$ levels of probability 
Table 10: Specific combining ability effects for each cross (Sij) and reciprocal effects (rij) for yield and its components over two seasons

\begin{tabular}{|c|c|c|c|c|c|c|}
\hline Crosses & $\begin{array}{c}\text { Plant } \\
\text { height }(\mathrm{cm})\end{array}$ & $\begin{array}{c}\text { Number of } \\
\text { branches }\end{array}$ & $\begin{array}{c}\text { Total } \\
\text { chlorophyll }\end{array}$ & $\begin{array}{l}\text { Early fruit } \\
\text { yield(kg) }\end{array}$ & number & $\begin{array}{c}\text { Total fruit } \\
\text { yield(ton/fed) }\end{array}$ \\
\hline $1 \times 2$ & $4.7625^{\star *}$ & $0.9438^{* *}$ & $-0.1262^{* \star}$ & $2.0629^{\star *}$ & 13.4896 & 6.5316 \\
\hline $1 \times 3$ & $2.0167^{*}$ & $0.6896^{\star *}$ & 0.0042 & $1.086^{* *}$ & 4.0938 & 1.0266 \\
\hline $1 \times 4$ & $-6.4^{\star \star}$ & $-1.1521^{\star *}$ & $0.1206^{\star \star}$ & $-1.4061^{\star *}$ & -2.1146 & -0.2181 \\
\hline $2 \times 1$ & -0.0667 & -0.0667 & $0.0726^{\star *}$ & $-0.552^{\star \star}$ & -5.25 & -0.8325 \\
\hline $2 \times 3$ & $5.4583^{* *}$ & 0.0813 & $0.065^{\star \star}$ & $-0.4215^{\star *}$ & 3.1354 & $22.4543^{\star \star}$ \\
\hline $2 \times 4$ & $-13.2583^{* *}$ & $-1.0271^{\star *}$ & $-0.2109^{\star *}$ & $-1.0891^{\star *}$ & -21.7396 & $-25.7921^{* *}$ \\
\hline $3 \times 1$ & -0.9833 & $0.4833^{\star *}$ & $0.1083^{\star \star}$ & $1.8784^{\star \star}$ & $26.1667^{*}$ & -0.5923 \\
\hline $3 \times 2$ & -0.9167 & 0.05 & -0.001 & $0.8537^{* *}$ & 9.6667 & $-7.9083^{*}$ \\
\hline $3 \times 4$ & $-11.1542^{\star *}$ & $-1.2646^{* *}$ & $-0.1337^{* *}$ & $-1.2655^{\star \star}$ & -24.7188 & $-22.2602^{\star \star}$ \\
\hline $4 \times 1$ & 0.2 & 0.0333 & $0.1187^{* *}$ & 0 & 0 & $-11.1901^{\star *}$ \\
\hline $4 \times 2$ & -0.3333 & $0.2667^{\star \star}$ & 0.0084 & 0 & 0 & 1.745 \\
\hline $4 \times 3$ & $-4.1333^{\star \star}$ & $-0.2667^{* *}$ & $-0.0442^{* *}$ & 0 & 0 & -0.4242 \\
\hline S.E(Sij) & 0.4795 & 0.1708 & 0.0465 & 0.1424 & 2.1218 & 1.1439 \\
\hline S.E(rij) & 0.3714 & 0.1323 & 0.0360 & 0.1103 & 1.6436 & 0.8861 \\
\hline
\end{tabular}

* Significant at $0.05 \%,{ }^{* \star}$ Significant at $0.01 \%$ levels of probability

Table 11: Specific combining ability effects for each cross (Sij) and reciprocal effects (rij) for chemical traits over two seasons

\begin{tabular}{|c|c|c|c|c|c|c|}
\hline Crosses & Total carotene & Anthocyanin content & Total nitrogen content & Total phosphorus & Potassium content & Total iron \\
\hline $1 \times 2$ & $-0.0011^{* \star}$ & $-3.5962^{* *}$ & $-0.3776^{* *}$ & $-0.008^{* *}$ & $-0.3305^{* *}$ & $0.8226^{* *}$ \\
\hline $1 \times 3$ & $-0.002^{* *}$ & $4.167^{\star \star}$ & 0.0382 & $0.0043^{\star *}$ & $-0.1374^{\star}$ & -0.0684 \\
\hline $1 \times 4$ & $0.0046^{* *}$ & $-3.5355^{* *}$ & $0.3712^{* *}$ & $0.0052^{* \star}$ & 0.042 & $0.3876^{* *}$ \\
\hline $2 \times 1$ & $-0.00067^{\star \star}$ & 0.0781 & $0.1258^{\star \star}$ & $-0.0043^{\star \star}$ & $-0.4267^{* *}$ & -0.0292 \\
\hline $2 \times 3$ & $0.0035^{* *}$ & $-1.2386^{* \star}$ & -0.0593 & $0.0177^{* *}$ & 0.1237 & $0.1668^{*}$ \\
\hline $2 \times 4$ & $0.0003^{* *}$ & $2.6965^{* *}$ & $0.1737^{* *}$ & $0.0002^{* *}$ & $0.1389^{*}$ & $-0.5730^{* *}$ \\
\hline $3 \times 1$ & $-0.0005^{\star *}$ & $1.1843^{\star \star}$ & $-0.3633^{\star *}$ & $-0.0271^{* \star}$ & 0.0875 & $0.4383^{\star *}$ \\
\hline $3 \times 2$ & $-0.00008^{\star \star}$ & $0.5179^{* \star}$ & 0.0108 & $-0.0048^{* \star}$ & $0.2817^{* *}$ & $-0.515^{* *}$ \\
\hline $3 \times 4$ & $0.0016^{* \star}$ & $-2.3488^{* *}$ & $0.2345^{* \star}$ & $0.0062^{* \star}$ & 0.0012 & $-1.0766^{* \star}$ \\
\hline $4 \times 1$ & $-0.0002^{* \star}$ & 0.141 & $-0.2033^{* *}$ & $-0.0078^{* \star}$ & -0.0525 & $1.5683^{* *}$ \\
\hline $4 \times 2$ & $-0.0006^{* *}$ & -0.0703 & $-0.3258^{\star \star}$ & $0.0066^{* *}$ & -0.0692 & $0.5825^{* *}$ \\
\hline $4 \times 3$ & $-0.0005^{* *}$ & $0.4207^{* *}$ & $0.3417^{* *}$ & $-0.0182^{* *}$ & $-0.3958^{* \star}$ & -0.0825 \\
\hline S.E(Sij) & 0.0007 & 0.2161 & 0.097279 & 0.0031 & 0.1318 & 0.1451 \\
\hline S.E(rij) & 0.0006 & 0.1674 & 0.0754 & 0.0024 & 0.1021 & 0.1124 \\
\hline
\end{tabular}

* Significant at $0.05 \%,{ }^{* *}$ Significant at $0.01 \%$ levels of probability

\section{REFERENCES}

Babu S., Chezhian P. and Ganesan J. (2000). Combining Ability Studies in Eggplant (Solanum melongena L,.). Tropical Agricultural Research Vol. 12:394-397.

Behera T.K., Singh Narendra (2002). Inter-specific crosses between eggplant (Solanum melongena L.) with related Solanum species. Scientia Horticulturae 95 : 165-172.

Brinda, N. and Sivasubramanian, V. (1993). Studies on combining ability reciprocal differences through diallel analysis in sesame. Plant Breeding Newsletter, 2.2.

Chowdhury M. J., S. Ahmad, M. Nazim Uddin, A. K. M. Quamruzzaman and M. M. A. Patwary(2010). Expression of Heterosis for Productive Traits in F1 Brinjal (Solanum melongena L.) Hybrids. 
A Scientific Journal of Krishi Foundation The Agriculturists 8(2): 8-13.

Cochran, W.G. and G.M. Cox (1957). Experimental Designs. $2^{\text {nd }}$ ed., John Willey and Sons, New York, USA. 611p.

Collonnier C, Fock I, Kashyap V, Rotino GL, Daunay MC, Lian Y, Mariska IK, Rajam MV, Servaes A, Ducreux G, Sihachakr D (2001) Applications of biotechnology in eggplant. Plant Cell, Tissue Organ Cult 65:91-107.

Griffing, B. (1956). Concept of general and specific combining ability in relation to diallel crossing systems. Australian. J. Biol. Sci., 9: 463-493.

Kumar Ajay, Bharat Bhushan, A.S. Sidhu and A.S. Dhatt (2012). Studies on combining ability for yield and quality traits in brinjal (Solanum melongena L.). J. Hortl. Sci. Vol. 7(2): 145-151.

Makani A. Y., A. L. Patel, M. M. Bhatt and P. C. Patel (2013). Heterosis for Yield and Its Contributing Attributes In Brinjal (Solanum melongena L.). An international quarterly journal of life science 8(4): 1369-1371.

Patil. S.A., Nalini A. Dharwad and Salimath P. M. (2011). Heterosis and combining ability analysis for productivity traits in brinjal (Solanum melongena L.). Karnataka J. Agric. Sci.,24 (5) : (622625) 2011.

Salama G.M., Melad. H. Z.; Faten and S. Saleeb (2005). Combining Ability and Correlation Between Yield and Different Characters in Eggplant for Producing High Quality of Local Hybrids. J. Agric. Sci. Mansoura Univ., 30 (1): 513-532.

Sharaf Uddin.M , M.M. Rahman, M.M. Hossain, M.A. Khaleque Mian(2015).

Combining Ability of Yield and Yield Components in Eggplant (Solanum melongena L.) During Summer. Universal Journal of Plant Science 3(4): 59-66.

Van Eck J and Snyder A. (2006). Eggplant (Solanum melongena L.). Methods Mol Biol.,343:439-47. 


\section{قوة الهجين والقدرة على التألف لبعض الصفات الناتجة عن التهجين داخل النوع النباتي وبين الأنواع في الباذنجان}

\section{محمد سعد حمادة1 , علي فتحي حمايل2 رسيف الدين محمد فريد3 ومها مجدي القاضي3}

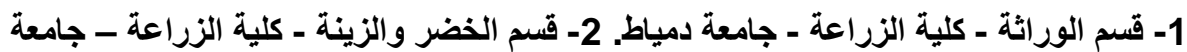

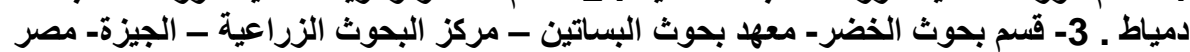

أجريت هذه الدراسة خلال الفترة من 2013 حتى والأى 2015 بهدف دراسة قوة الهجين

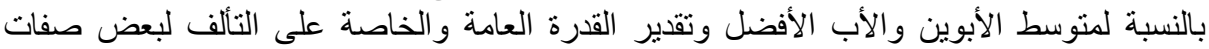

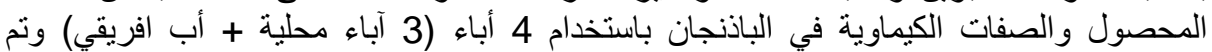

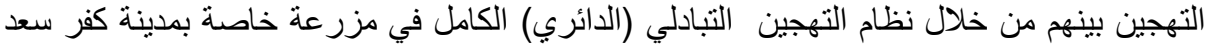

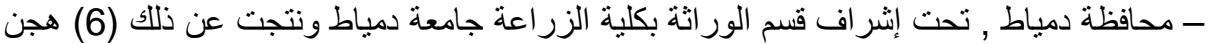
و) وجن عكسية . ظهرت قوة الهجين بالنسبة لمتوسط الأبوين والأب الأفضل لصفات طول النبات, عدد الكبر

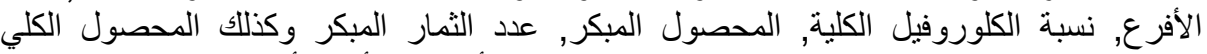

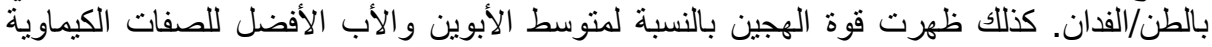

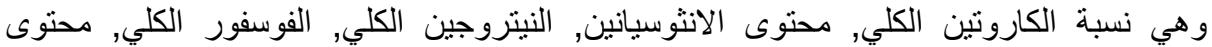

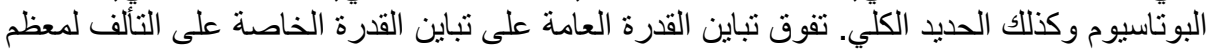

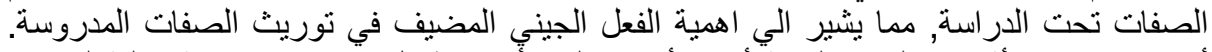

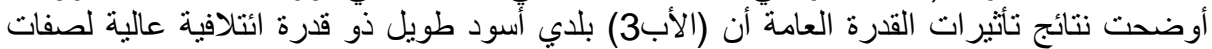

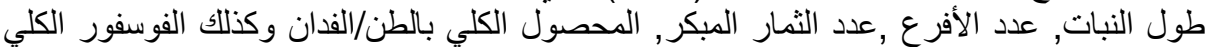

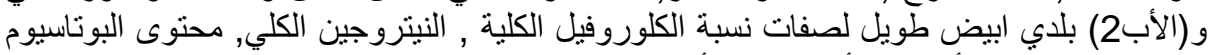

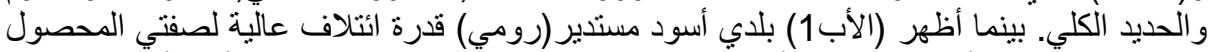

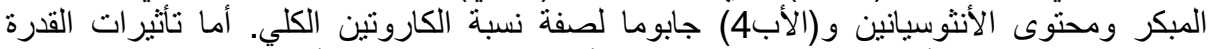

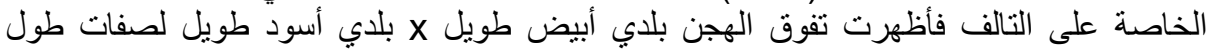

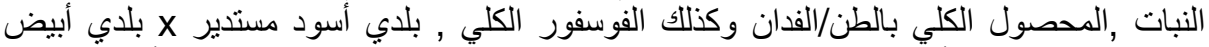

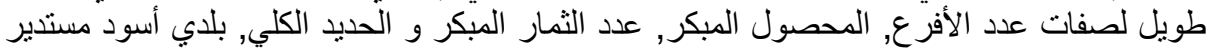

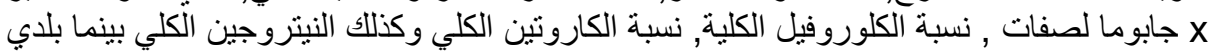

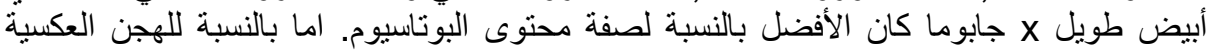

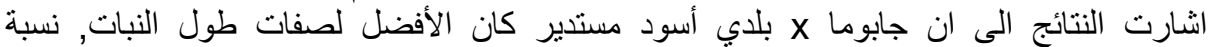

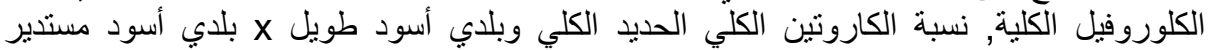

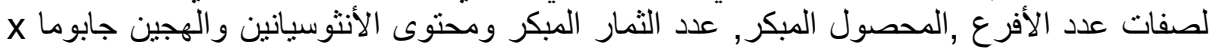

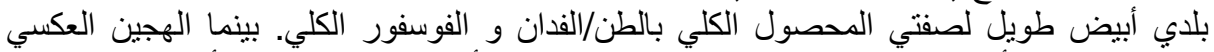

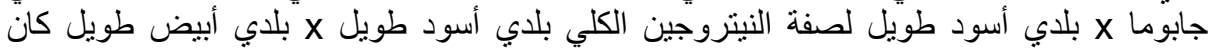

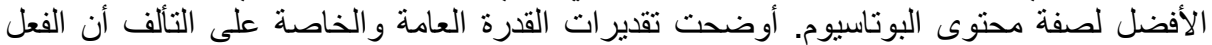

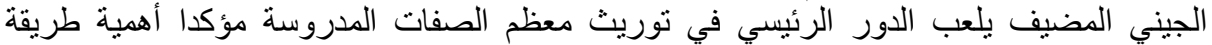

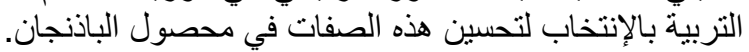

